\title{
PIECEWISE LINEAR APPROXIMATION OF LIPEOMORPHISMS
}

\author{
JUSSI VÄISÄLÄ
}

\section{Introduction}

1.1. A map $f$ of a metric space $(X, d)$ into a metric space $\left(Y, d^{\prime}\right)$ is called Lipschitz if there is a constant $L$ such that $d^{\prime}(f(x), f(y)) \leqq L d(x, y)$ for all $x, y$ in $X$. The smallest $L \geqq 0$ satisfying this inequality is denoted by $\operatorname{lip} f$. If every point in $X$ has a neighborhood in which $f$ is Lipschitz, $f$ is said to be locally Lipschitz, abbreviated LIP. A lipeomorphism is a bijective map $f$ such that both $f$ and $f^{-\mathbf{1}}$ are LIP. If $f$ defines a lipeomorphism $f_{1}: X \rightarrow f X, f$ is a LIP embedding. If both $f$ and $f_{1}^{-1}$ are Lipschitz, we set $\operatorname{bilip} f=\max \left(\operatorname{lip} f, \operatorname{lip} f_{1}^{-1}\right)$. A LIP map of a compact space is always Lipschitz. For the elementary theory of Lipschitz topology, we refer to [3].

1.2. Outline of results. Let $I^{n}$ be the closed unit cube in $R^{n}, n \leqq 3$, and let $f: I^{n} \rightarrow R^{n}$ be a LIP embedding. We shall prove that for every $\varepsilon>0$ there is a LIP embedding $g: I^{n} \rightarrow R^{n}$ such that $g\left|\partial I^{n}=f\right| \partial I^{n}, g \mid$ int $I^{n}$ is PL, and $|g(x)-f(x)|<\varepsilon$ for all $x$. Moreover, bilip $g$ is bounded by a constant depending only on $\operatorname{bilip} f, \varepsilon$, and $n$. Actually, it does not depend on $\varepsilon$ (see Remark 2.12), but $\varepsilon$ does not play any role in the applications. The proof is based on the clever ideas of Carleson [1]. As applications, we shall prove the LIP annulus conjecture for $n \leqq 3$ and the LIP hauptvermutung for $n=2$. D. Sullivan [7] has proved these results for more general $n$ by different methods.

1.3. Acknowledgement. I have greatly profited from the detailed seminar lectures of J. Sarvas on Carleson's method.

\section{Approximation}

2.1. Notation. We let $I^{n}$ denote the cube $[-1,1]^{n}$. If $a>0$, the cube $a I^{n}$ can also be written as $I^{n}(a)$. Let $K^{*}$ be the family of $4^{n}-2^{n}$ closed cubes of side length one, giving the natural subdivision of $2 I^{n} \backslash$ int $I^{n}$. If $f: X \rightarrow R^{n}$ is a map and if $A \subset X$, we write $\|f\|_{A}=\sup \{|f(x)|: x \in A\}$ and $\|f\|=\|f\|_{X}$.

2.2. Lemma. Let $n=2$ or 3 and let $P$ be the union of a subfamily of $K^{*}$ such that $P$ and $I^{n} \cup P$ are $n$-manifolds and $I^{n} \cap P$ is an $(n-1)$-manifold. Let $\varepsilon>0$ 
and let $g: 2 I^{n} \rightarrow R^{n}$ be an embedding. Then there is $\delta>0$ with the following property. If $g_{1}: P \rightarrow R^{n}$ is a PL embedding with $\left\|g_{1}-g\right\|_{P}<\delta$, there is a PL embedding $g^{*}: I^{n} \cup$ $\cup P \rightarrow R^{n}$ such that $\left\|g^{*}-g\right\|<\varepsilon$ and $g^{*} \mid P=g_{1}$.

2.3. If $n=3$, the lemma follows from a more general and stronger result of Moise [4, p. 101]. The proof for $n=2$ is simpler.

The lemma is not true for $n \geqq 5$, because it would yield the hauptvermutung for compact PL manifolds. Indeed, we could apply it to a fine handle decomposition of a PL manifold and obtain a PL approximation to every homeomorphism between PL manifolds. Thus the method of this paper cannot be directly extended to higher dimensions.

The rest of this section is devoted to the proof of the following result:

2.4. Theorem. Let $n \leqq 3$, let $\varepsilon>0$, and let $L \geqq 1$. Then there is $L_{1} \geqq 1$ with the following property: If $f: I^{n} \rightarrow R^{n}$ is a LIP embedding with bilip $f \leqq L$, there is a LIP embedding $f^{*}: I^{n} \rightarrow R^{n}$ such that

(1) $f^{*}\left|\partial I^{n}=f\right| \partial I^{n}$

(2) $f^{*} \mid$ int $I^{n}$ is PL,

(3) $\operatorname{bilip} f^{*} \leqq L_{1}$,

(4) $\left\|f^{*}-f\right\|<\varepsilon$.

2.5. If $n=1$, Theorem 2.4 is almost trivial. For the rest of this section we fix $n \in\{2,3\}$. Given $\varepsilon$ and $g$ as in 2.2 , we let $\delta(\varepsilon, g)$ denote the greatest $\delta$ satisfying 2.2 for all $P$. Obviously $\delta(\varepsilon, g) \leqq \varepsilon$. Let $H_{L}$ be the space of all LIP embeddings $g: 2 I^{n} \rightarrow R^{n}$ such that $|g(0)| \leqq 1$ and bilip $g \leqq L$. In the uniform topology, $H_{L}$ is closed in the space of all continuous maps $2 I^{n} \rightarrow R^{n}$. Since $H_{L}$ is equicontinuous and uniformly bounded, it is compact by Ascoli's theorem. We set $\delta^{*}(\varepsilon)=$ $=\inf \left\{\delta(\varepsilon, g) \mid g \in H_{L}\right\}$.

\subsection{Lemma. $\delta^{*}(\varepsilon)>0$ for every $\varepsilon>0$.}

Proof. If the lemma is false, there is a sequence of maps $g_{j} \in H_{L}$ such that $\delta\left(\varepsilon, g_{j}\right) \rightarrow 0$. By the compactness of $H_{L}$, we may assume that $g_{j} \rightarrow g \in H_{L}$. If $2\left\|g_{j}-g\right\|<\delta(\varepsilon / 2, g)$, then 2.2 holds with the substitution $g \mapsto g_{j}, \delta \mapsto \delta(\varepsilon / 2, g) / 2$. Hence $\delta\left(\varepsilon, g_{j}\right) \geqq \delta(\varepsilon / 2, g)>0$ for large $j$, which is a contradiction.

2.7. The family $K$. We divide int $I^{n}$ into a countable family $K$ of closed cubes as follows: First let $I^{n}(1 / 3) \in K$. Next divide $I^{n}(2 / 3) \backslash$ int $I^{n}(1 / 3)$ in the natural way into $4^{n}-2^{n}$ cubes of side length $1 / 3$. In the general step, we divide $I^{n}\left(1-3^{-1} 2^{-k-1}\right) \backslash$ int $I^{n}\left(1-3^{-1} 2^{-k}\right)$ into cubes of side length $3^{-1} 2^{-k-1}$.

Each $Q \in K$ can be uniquely written as $z_{Q}+\lambda_{Q} I^{n}$. Here $z_{Q}$ is the center and $2 \lambda_{Q}$ the side length of $Q$. Setting $\alpha_{Q}(x)=z_{Q}+\lambda_{Q} x$ we have $Q=\alpha_{Q} I^{n}$.

We express $K$ as the union of a finite number of disjoint subfamilies $K_{1}, \ldots, K_{M}$ such that setting $K_{r}^{*}=K_{1} \cup \ldots \cup K_{r}, F_{r}=\cup\left\{Q \mid Q \in K_{r}^{*}\right\}$ and $P_{Q}=2 I^{n} \cap \alpha_{Q}^{-1} F_{r-1}$ for $Q \in K_{r}$, the following conditions are satisfied: 
(1) The members of $K_{r}$ are pairwise disjoint.

(2) $P_{Q}$ and $I^{n} \cup P_{Q}$ are $n$-manifolds, and $I^{n} \cap P_{Q}$ is an (n-1)-manifold.

(3) If $Q, R \in K_{r}$, then $P_{Q}=P_{R}$.

It is obvious that this can be done. In the figure we indicate this for $n=2$ with $M=22$.

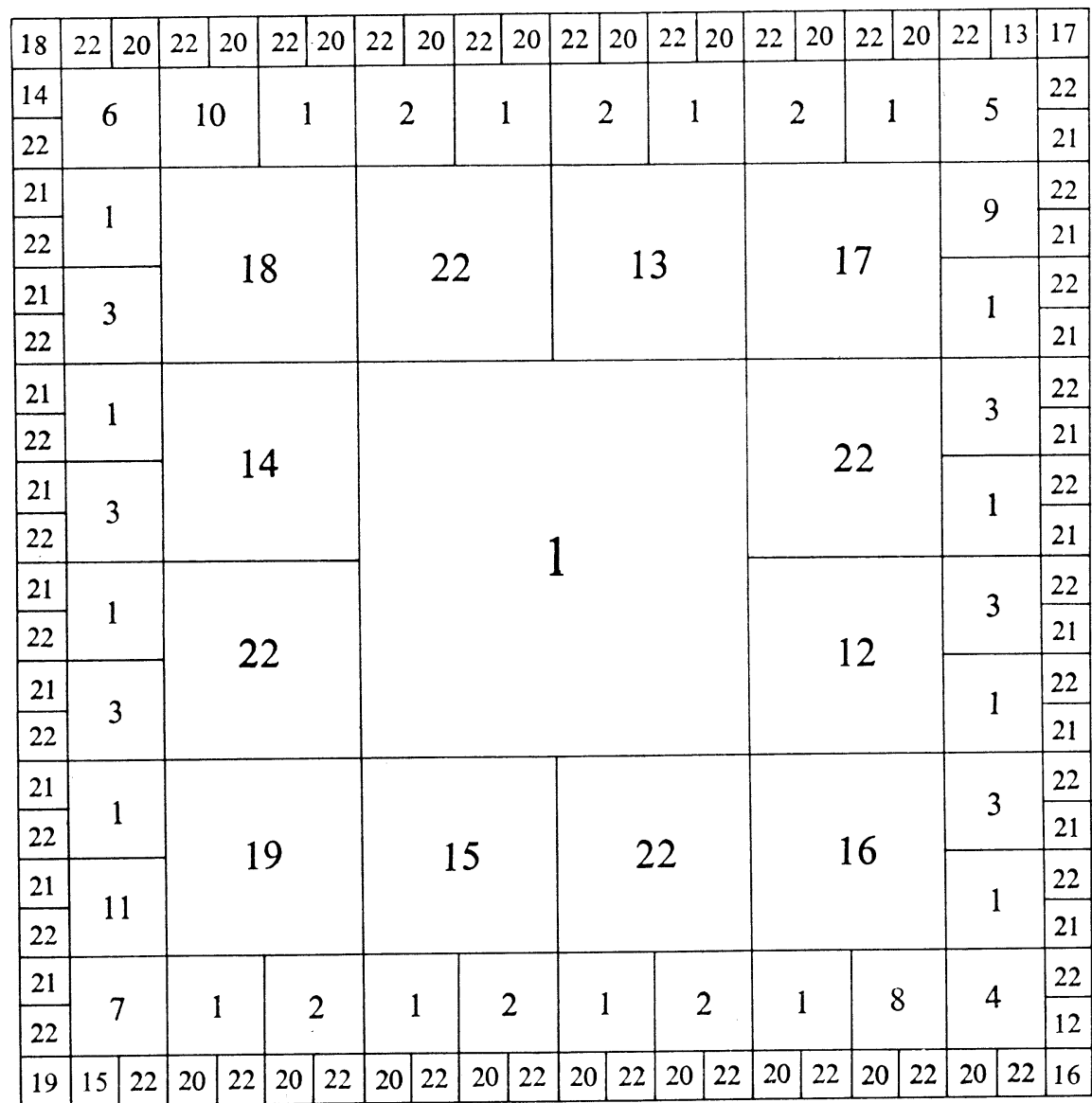

2.8. For the rest of the proof we fix $\varepsilon>0$ and $L \geqq 1$. Let $M$ be the integer given by 2.7. Choose positive numbers $\delta_{1} \leqq \ldots \leqq \delta_{M}$ such that $\delta_{M} \leqq \min (\varepsilon, 1 / 8 L)$ and $5 \delta_{r-1}<\delta^{*}\left(\delta_{r}\right)$. Observe that the numbers $\delta_{r}$ only depend on $\varepsilon$ and $L$ (and $n$ ).

Suppose now that $f: I^{n} \rightarrow R^{n}$ is a LIP embedding with bilip $f \leqq L$. For every $Q \in K$ choose a point $b_{Q} \in \lambda_{Q} Z^{n}$ such that $\left|f\left(z_{Q}\right)-b_{Q}\right|<\lambda_{Q}$. Define $\beta_{Q}: R^{n} \rightarrow R^{n}$ by $\beta_{Q}(x)=\left(x-b_{Q}\right) / \lambda_{Q}$. 
2.9. Lemma. There are finite families $A_{r}(L, \varepsilon), 1 \leqq r \leqq M$, of PL embeddings $I^{n} \rightarrow R^{n}$ such that if $f: I^{n} \rightarrow R^{n}$ is a LIP embedding with bilip $f \leqq L$, there are injective PL maps $\varphi_{r}: F_{r} \rightarrow R^{n}$ such that

(i) $\beta_{Q} \varphi_{r} \alpha_{Q} \mid I^{n} \in A_{r}(L, \varepsilon)$ for every $Q \in K_{r}^{*}$,

(ii) $\left\|f-\varphi_{r}\right\|_{Q} \leqq 2 \delta_{r} \lambda_{Q}$ for every $Q \in K_{r}^{*}$.

2.10. Proof of 2.4 modulo 2.9. Since $F_{M}=\operatorname{int} I^{n}$, we obtain a function $f^{*}: I^{n} \rightarrow R^{n}$ by setting $f^{*}=\varphi_{M} \cup\left(f \mid \partial I^{n}\right)$. If $a>0$, the set $U(a)=\left(\partial I^{n}+a I^{n}\right) \cap i n t I^{n}$ is contained in the union of those $Q \in K$ for which $\lambda_{Q}<a$. Hence (ii) implies $\left\|f-f^{*}\right\|_{U(a)} \leqq$ $\leqq 2 \delta_{M} a$, which proves the continuity of $f^{*}$. Moreover, $f^{*}$ is an embedding, $\operatorname{im} f^{*}=$ $=\operatorname{im} f$, and $f^{*} \mid \operatorname{int} I^{n}=\varphi_{M}$ is PL. Since $\left\|f^{*}-f\right\|=\sup _{Q}\left\|f-\varphi_{M}\right\|_{Q} \leqq 2 \delta_{M} \sup _{Q} \lambda_{Q}=$ $=2 \delta_{M} / 3<\varepsilon$, it remains to show that bilip $f^{*}$ has an upper bound $L_{1}$ which only depends on $L, \varepsilon$, and $n$. Since $f^{*} \mid Q=\beta_{Q}^{-1} \psi \alpha_{Q}^{-1}$ for some $\psi \in A_{M}(L, \varepsilon)$ and since $I^{n}$ is convex, $\operatorname{lip} f^{*} \leqq \max \left\{\operatorname{bilip} \psi \mid \psi \in A_{M}(L, \varepsilon)\right\}=L_{0}$ [3, 2.35]. Since $f^{*}\left[\right.$ int $\left.I^{n}\right]=$ $=f$ [int $\left.I^{n}\right]$ is $L^{2}$-quasiconvex, $\operatorname{lip}\left(f^{*}\right)^{-1} \leqq L^{2} L_{0}$. Thus bilip $f^{*} \leqq L^{2} L_{0}$.

2.11. Proof of 2.9. We shall inductively construct the sets $A_{r}(L, \varepsilon)$ and the maps $\varphi_{r}$ such that (i) and (ii) are satisfied. Since $H_{L}$ is compact, there is a finite set $H(L, \varepsilon) \subset H_{L}$ such that $H_{L}$ is covered by the open balls $\left\{h \in H_{L}:\|h-g\|<\delta_{1}\right\}$, $g \in H(L, \varepsilon)$. For every $h \in H(L, \varepsilon)$ we choose a PL embedding $g_{h}: I^{n} \rightarrow R^{n}$ such that $\left\|g_{h}-h\right\|_{I^{n}}<\delta_{1}\left[5\right.$, p. 239] and set $A_{1}(L, \varepsilon)=\left\{g_{h} \mid h \in H(L, \varepsilon)\right\}$. For every $Q \in K$, the map $f_{Q}=\beta_{Q} f \alpha_{Q} \mid 2 I^{n}$ belongs to $H_{L}$, since $|f(0)|=\left|f\left(z_{Q}\right)-b_{Q}\right| / \lambda_{Q}<1$. Choose maps $h_{Q} \in H(L, \varepsilon)$ such that $\left\|h_{Q}-f_{Q}\right\|<\delta_{1}$ and set $g_{Q}=g_{h_{Q}}$. Then $\left\|f_{Q}-g_{Q}\right\|_{I^{n}}<2 \delta_{1}$. Define $\varphi_{1}: F_{1} \rightarrow R^{n}$ by $\varphi_{1} \mid Q=\beta_{Q}^{-1} g_{Q} \alpha_{Q}^{-1}$ for $Q \in K_{1}$. Then $\varphi_{1}$ is PL, (i) is true, and (ii) follows from $\left\|f-\varphi_{1}\right\|_{Q}=\lambda_{Q}\left\|f_{Q}-g_{Q}\right\|_{I^{n}}$. To show that $\varphi_{1}$ is injective, suppose that $Q$ and $R$ are two cubes in $K_{1}$. Assuming $\lambda_{Q} \geqq \lambda_{R}$ we have $d(Q, R) \geqq \lambda_{Q}$, which implies $d(f Q, f R) \geqq \lambda_{Q} / L$. Since $\delta_{1} \leqq \delta_{M} \leqq 1 / 8 L$, (ii) implies $d\left(\varphi_{1} Q, \varphi_{1} R\right) \geqq$ $\geqq \lambda_{Q} / 2 L>0$.

Suppose now that $A_{r-1}(L, \varepsilon)$ and $\varphi_{r-1}: F_{r-1} \rightarrow R^{n}$ have been constructed so that (i) and (ii) are satisfied. Let $Q \in K_{r}$. The polyhedron $P=P_{Q}$ is independent of $Q$ by the condition (3) of 2.7. Set $\gamma_{Q}=\beta_{Q} \varphi_{r-1} \alpha_{Q} \mid P$. If $x \in P$, there is $R \in K_{r-1}^{*}$ such that $\alpha_{Q}(x) \in R$ and $Q \cap R \neq \emptyset$. Then $\gamma_{Q}(x)=\beta_{Q} \beta_{R}^{-1} \psi \alpha_{R}^{-1} \alpha_{Q}(x)$, where $\psi=\beta_{R} \varphi_{r-1} \alpha_{R} \mid I^{n} \in A_{r-1}(L, \varepsilon)$ and

$$
\begin{aligned}
& \alpha_{R}^{-1} \alpha_{Q}(x)=\lambda_{Q} x / \lambda_{R}+\left(z_{Q}-z_{R}\right) / \lambda_{R}, \\
& \beta_{Q} \beta_{R}^{-1}(y)=\lambda_{R} y / \lambda_{Q}+\left(b_{R}-b_{Q}\right) / \lambda_{Q} .
\end{aligned}
$$

Since $Q \cap R \neq \emptyset, \lambda_{Q} / \lambda_{R} \in\{1 / 2,1,2\}$ and $\left(z_{Q}-z_{R}\right) / \lambda_{R}$ also belongs to a finite set depending only on $n$. Hence the maps $\alpha_{R}^{-1} \alpha_{Q}$ belong to a finite set $A$. Furthermore, since $\left|b_{R}-b_{Q}\right| \leqq\left|b_{R}-f\left(z_{R}\right)\right|+\left|f\left(z_{R}\right)-f\left(z_{Q}\right)\right|+\mid f\left(z_{Q}-b_{Q} \mid<(3+6 L) \lambda_{Q}\right.$, the maps $\beta_{Q} \beta_{R}^{-1}$ belong to a finite set $B(L)$. Hence the maps $\gamma_{Q}$ belong to a finite set $C_{r}(L, \varepsilon)$.

We want to apply Lemma 2.2 with the substitution $P \mapsto P, \varepsilon \mapsto \delta_{r}, g_{\mapsto} \mapsto \dot{r}_{Q} \mid I^{n} \cup P$, $g_{1} \mapsto \gamma_{Q}$. We must verify the condition $\left\|\gamma_{Q}-h_{Q}\right\|_{P}<\delta^{*}\left(\delta_{r}\right)$. Let $x \in P$ and choose $R$ as above. Then $\left|\gamma_{Q}(x)-f_{Q}(x)\right|=\left|\varphi_{r-1}\left(\alpha_{Q}(x)\right)-f\left(\alpha_{Q}(x)\right)\right| / \lambda_{Q} \leqq 2 \delta_{r-1} \lambda_{R} / \lambda_{Q} \leqq 4 \delta_{r-1}$, which implies $\left\|\gamma_{Q}-h_{Q}\right\|_{P} \leqq 5 \delta_{r-1}<\delta^{*}\left(\delta_{r}\right)$. 
By 2.2, there is a PL embedding $g_{Q}^{*}: I^{n} \cup P \rightarrow R^{n}$ such that $g_{Q}^{*} \mid P=\gamma_{Q}$ and $\left\|h_{Q}-g_{Q}^{*}\right\|_{I^{n_{U} P}}<\delta_{r}$. Since the pairs $\left(h_{Q}, \gamma_{Q}\right)$ belong to the finite set $H(L, \varepsilon) \times C_{r}(L, \varepsilon)$, we can choose the maps $g_{Q}^{*}$ so that they belong to a finite set $G_{r}(L, \varepsilon)$. Define $\varphi_{r}: F_{r} \rightarrow R^{n}$ as follows:

$$
\begin{gathered}
\varphi_{r} \mid F_{r-1}=\varphi_{r-1}, \\
\varphi_{r}\left|Q=\beta_{Q}^{-1} g_{Q}^{*} \alpha_{Q}^{-1}\right| Q \text { for } Q \in K_{r} .
\end{gathered}
$$

It is clear that $\varphi_{r}$ is a well-defined PL map. The condition (i) is satisfied with $A_{r}(L, \varepsilon)=$ $=A_{r-1}(L, \varepsilon) \cup G_{r}(L, \varepsilon)$. If $Q \in K_{r}$, then $\left\|f-\varphi_{r}\right\|_{Q}=\lambda_{Q}\left\|f_{Q}-g_{Q}^{*}\right\|_{I^{n}}<2 \lambda_{Q} \delta_{r}$, which is (ii). The restrictions of $\varphi_{r}$ to $F_{r-1}$ and $\alpha_{Q}\left[I^{n} \cup P\right], Q \in K_{r}$, are injective. To show that $\varphi_{r}$ is injective, it suffices to prove that $\Delta=\left|\varphi_{r}(x)-\varphi_{r}(y)\right|>0$ whenever $x \in Q \in K_{r}$ and $y \in F_{r} \backslash \alpha_{Q}\left[I^{n} \cup P\right]$. Choosing $R \in K_{r}^{*}$ containing $y$ we obtain $\Delta \geqq|x-y| / L-$ $-2 \delta_{r}\left(\lambda_{Q}+\lambda_{R}\right)$ and $|x-y| \geqq \lambda_{Q}$. If $\lambda_{R} \leqq 2 \lambda_{Q}$, then $\Delta \geqq \lambda_{Q} / 4 L$. If $\lambda_{R}>2 \lambda_{Q}$, then $Q \cap R=\emptyset$, whence $|x-y| \geqq \lambda_{R}$ and $\Delta \geqq 5 \lambda_{R} / 8 L$.

2.12. Remarks. 1 . One can prove that the constant $L_{1}$ in Theorem 2.4 only depends on $L$ and $n$, not on $\varepsilon$. This is done by replacing the cube family $K$ by a suitable subdivision of small mesh. For example, we may bisect the sides of each cube in $K$ and iterate this process. One must only verify that there are subfamilies $K_{1}^{\prime}, \ldots, K_{M}^{\prime}$ of $K^{\prime}$ as in 2.7 such that $M$ is independent of $K^{\prime}$.

2. A direct application of Carleson's method also gives the following result: For every $n \leqq 3$ and $L \geqq 1$ there is $L_{1} \geqq 1$ such that if $f: R^{n} \rightarrow R^{n}$ is a strong lipeomorphism with bilip $f \leqq L$ and if $\varepsilon>0$, there is a PL homeomorphism $f^{*}: R^{n} \rightarrow R^{n}$ such that $\left\|f^{*}-f\right\|<\varepsilon$ and bilip $f^{*} \leqq L_{1}$.

3. A quasiconformal version of 2.4 has been recently proved by M. Kiikka.

\section{Applications}

3.1. LIP stability. A lipeomorphism $f: R^{n} \rightarrow R^{n}$ is called LIP stable if it can be expressed as $f_{1} \ldots f_{k}$, where each $f_{j}: R^{n} \rightarrow R^{n}$ is a lipeomorphism which is the identity map on some non-empty open set. If $f$ is LIP stable and if $g: R^{n} \rightarrow R^{n}$ is a lipeomorphism agreeing with $f$ on some non-empty open set, then $g$ is also LIP stable.

Suppose that $n \leqq 3$ and $f: R^{n} \rightarrow R^{n}$ is an arbitrary lipeomorphism. It follows from 2.4 that there is a lipeomorphism $g: R^{n} \rightarrow R^{n}$ such that $f=g$ in $R^{n} \backslash I^{n}$ and $g \mid$ int $I^{n}$ is PL. An elementary argument shows that an affine map with positive determinant is LIP stable. Hence we obtain:

3.2. Theorem. If $n \leqq 3$, every sense-preserving lipeomorphism $f: R^{n} \rightarrow R^{n}$ is LIP stable.

3.3. The LIP annulus conjecture states that if $S_{1}$ and $S_{2}$ are disjoint locally LIP flat LIP $(n-1)$-spheres in $S^{n}$, [3, 3.8], then the closure of the domain bounded by $S_{1} \cup S_{2}$ is lipeomorphic to $S^{n-1} \times I$. Or equivalently, there is a lipeomorphism 
$S^{n} \rightarrow S^{n}$ mapping $S_{1}$ and $S_{2}$ onto round spheres. In the topological category, the annulus conjecture follows from the TOP version of 3.2. This proof [2] is based on the Schönflies theorem, which is also true in LIP, $[3,7.8]$. Hence it can be rewritten in LIP, and we obtain:

3.4. Theorem. The LIP annulus conjecture is true for $n \leqq 3$.

3.5. A metric space $J$ is a LIP arc, half string or string if it is lipeomorphic to a closed, half open or open interval, respectively. If $J \subset R^{n}$, it is called locally LIP flat if each point of $J$ has a neighborhood $U$ such that $(U, U \cap J)$ is lipeomorphic to $\left(R^{n}, R^{1}\right)$ or $\left(R^{n}, R_{+}^{1}\right)$. In this case, there is a neighborhood $G$ of $J$ such that $(G, J)$ is lipeomorphic to $\left(R^{n}, I^{1}\right),\left(R^{n}, R_{+}^{1}\right)$ or $\left(R^{n}, R^{1}\right)$. This is seen by rewriting the corresponding TOP proofs $[6,3.4]$ in the LIP category. This justifies the abbreviation "LIP flat" for "locally LIP flat LIP" arcs, half strings and strings. If $J \subset R^{n}$ is a LIP flat arc, it follows from the LIP Schönflies theorem that $\left(R^{n}, J\right)$ is lipeomorphic to $\left(R^{n}, I^{1}\right)$.

3.6. Theorem. Let $n=2$ or 3 and let $G \subset R^{n}$ be a domain containing sets $J_{1}, J_{2}$ each of which is a LIP flat arc or half string, closed in G. If $P$ is an end point of $J_{1}$ and $Q$ an end point of $J_{2}$, there is $a$ LIP flat arc $J \subset G$ joining $P$ and $Q$ such that $J_{1} \cup J \cup J_{2}$ is a LIP flat arc, half string or string.

Proof. Choose an open LIP $n$-ball $D \subset G$ containing $P$ and $Q$ such that $D \backslash\left(J_{1} \cup J_{2}\right)$ is connected. For $n=3$ the latter condition is automatically true. For $n=2$ we can use the fact that an open TOP disk in $R^{2}$ is also an open LIP disk by the Riemann mapping theorem or by [3, 8.4]. Let $g: D \rightarrow R^{n}$ be a lipeomorphism. Set $J_{i}^{*}=g\left[D \cap J_{i}\right]$. We may assume that $J_{1}^{*}$ is a line segment near $g(P)$. There is a sense-preserving lipeomorphism $f: R^{n} \rightarrow R^{n}$ such that $f J_{2}^{*}$ is a line segment near $f(g(Q))$. By 3.2, $f$ can be written as $f_{k} \ldots f_{1}$, where each $f_{j}: R^{n} \rightarrow R^{n}$ is a lipeomorphism and $f_{j} \mid U_{j}=\mathrm{id}$ for some open $U_{j} \neq \emptyset$. (Actually, it is possile to choose $k=2$, which would slightly simplify the proof.) Choose a PL arc $A_{1}$ joining $g(P)$ to a point $y_{1} \in U_{1}$ such that $A_{1} \cap J_{1}^{*}=g(P)$ and $A_{1} \cap J_{2}^{*}=\emptyset$. Next choose a PL $\operatorname{arc} A_{2}$ joining $y_{1}$ to $y_{2} \in U_{2}$ such that $A_{2} \cap f_{1}\left[J_{1}^{*} \cup A_{1}\right]=y_{1}$ and $A_{2} \cap f_{1} J_{2}^{*}=\emptyset$. Continuing similarly we obtain PL arcs $A_{3}, \ldots, A_{k}$ with end points $y_{j} \in U_{j}$. Finally join $y_{k}$ to $f(g(Q))$ by a PL arc $A_{k+1}$ which does not meet the sets $f J_{1}^{*}, f J_{2}^{*}$ and $J^{*}=$ $=f A_{1} \cup f_{k} \ldots f_{2} A_{2} \cup \ldots \cup f_{k} A_{k}$ except at the end points. Then $J=g^{-1} f^{-1}\left[J^{*} \cup A_{k+1}\right]$ ist the desired arc.

\subsection{Theorem. Every LIP 2-manifold is lipeomorphic to a PL manifold.}

Proof. Let $M$ be a LIP 2-manifold. Then there is a PL manifold $P$ and a homeomorphism $f: P \rightarrow M$. Choose a triangulation $K$ of $P$ such that for every simplex $\sigma \in K, f \sigma$ is contained in an open LIP disk or half disk $U(\sigma)$. For the vertices $v$ of $K$ we can choose $U(v)$ so that $f^{-1} U(v) \subset$ st $(v, K)$ and the sets $U(v)$ are disjoint. Let $g$ be a lipeomorphism of $U(v)$ onto $R^{2}$ or $R_{+}^{2}$ such that $g(f(v))=0$. Let $\tau$ be 
a 1-simplex having $v$ as a vertex. Let $b(v, \tau)$ be the last point of $f \tau$ lying in $g^{-1} I^{2}$, and let $A(v, \tau)$ be the line segment joining 0 and $g(b(v, \tau))$. Then $J(v, \tau)=g^{-1} A(v, \tau)$ is a LIP flat arc in $U(v)$.

Let $\tau$ be an arbitrary 1-simplex of $K$, not lying on $\partial P$. It has two vertices $v_{1}$, $v_{2}$, and is a face of two 2-simplexes $\sigma_{1}, \sigma_{2}$. Set $b_{i}=b\left(v_{i}, \tau\right)$, and let $\tau_{0}$ be the subarc of $f \tau$ having end points $b_{i}$. Choose an open neighborhood $V(\tau)$ of $\tau_{0}$ in $U\left(\sigma_{1}\right) \cap$ $\cap U\left(\sigma_{2}\right) \cap\left(f \sigma_{1} \cup f \sigma_{2}\right)$ such that the sets $V(\tau)$ are disjoint. By 3.6, we can join $b_{1}$ and $b_{2}$ in $V(\tau)$ by an arc which together with $J\left(v_{1}, \tau\right)$ and $J\left(v_{2}, \tau\right)$ forms a LIP flat arc $A(\tau)$ whose end points are $f\left(v_{1}\right)$ and $f\left(v_{2}\right)$.

If $\tau \subset \partial P$, we set $A(\tau)=f \tau$. For every 1 -simplex $\tau$, we choose a lipeomorphism $h: \tau \rightarrow A(\tau)$ such that $h|\partial \tau=f| \partial \tau$. These maps give a LIP embedding $h:\left|K^{1}\right| \rightarrow M$. If $\sigma$ is a 2 -simplex of $K, h \partial \sigma$ is a locally LIP flat LIP circle in $U(\sigma)$. By the LIP Schönflies theorem, $h \mid \partial \sigma$ can be extended to a LIP embedding $\sigma \rightarrow M$, and we obtain a lipeomorphism $P \rightarrow M$.

3.8. Theorem (Lipvermutung, $n=2$ ). If $M_{1}, M_{2}$ are homeomorphic LIP 2-manifolds, they are lipeomorphic.

Proof. By 3.7, $M_{i}$ is lipeomorphic to a PL manifold $P_{i}$. By the classical 2-dimensional hauptvermutung, $P_{1}$ and $P_{2}$ are PL homeomorphic, hence lipeomorphic.

\section{References}

[1] Carleson, L.: The extension problem for quasiconformal mappings. - Contributions to Analysis, edited by L. V. Ahlfors et al., Academic Press, New York-London, 1974, 39—47.

[2] KIRBy, R. C.: Lectures on triangulations of manifolds. - Mimeographed notes, UCLA, 1969.

[3] Luukkainen, J., and J. VÄIsÄlä: Elements of Lipschitz topology. - Ann. Acad. Sci. Fenn. Ser. A I 3, 1977, 85-122.

[4] Moise, E. E.: Affine structures in 3-manifolds V. The triangulation theorem and Hauptvermutung. - Ann. of Math. 56, 1956, 96-114.

[5] Moise, E. E.: Geometric topology in dimension 2 and 3. - Springer-Verlag, New York-Heidelberg-Berlin, 1977.

[6] Rushing, T. B.: Topological embeddings. - Academic Press, New York-London, 1973.

[7] Sullivan, D.: Hyperbolic geometry and homeomorphisms. - To appear in the Proceedings of the Georgia Topology Conference 1977.

University of Helsinki

Department of Mathematics

SF-00100 Helsinki 10

Finland

Received 9 February 1978 